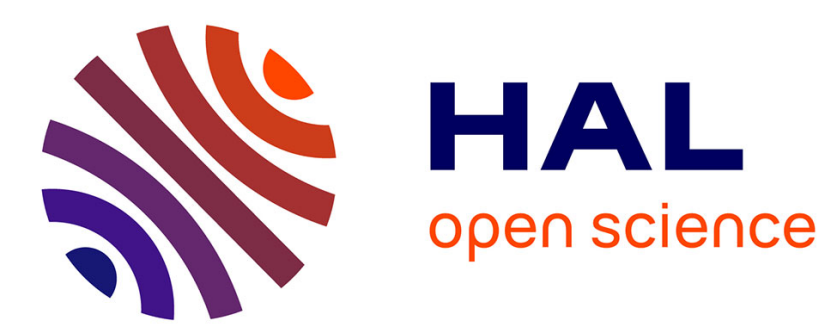

\title{
A nuclear field treatment of the 3-particle system outside closed-shell nuclei
}

\author{
R.J. Liotta, B. Silvestre-Brac
}

\section{To cite this version:}

R.J. Liotta, B. Silvestre-Brac. A nuclear field treatment of the 3-particle system outside closed-shell nuclei. Journal de Physique Lettres, 1978, 39 (2), pp.11-14. 10.1051/jphyslet:0197800390201100 . jpa-00231431

\section{HAL Id: jpa-00231431 https://hal.science/jpa-00231431}

Submitted on 1 Jan 1978

HAL is a multi-disciplinary open access archive for the deposit and dissemination of scientific research documents, whether they are published or not. The documents may come from teaching and research institutions in France or abroad, or from public or private research centers.
L'archive ouverte pluridisciplinaire HAL, est destinée au dépôt et à la diffusion de documents scientifiques de niveau recherche, publiés ou non, émanant des établissements d'enseignement et de recherche français ou étrangers, des laboratoires publics ou privés. 


\title{
LE JOURNAL DE PHYSIQUE-LETTRES
}

\author{
Classification \\ Physics Abstracts
}

$21.60 \mathrm{E}$

\section{A NUCLEAR FIELD TREATMENT OF THE 3-PARTICLE SYSTEM OUTSIDE CLOSED-SHELL NUCLEI}

\author{
R. J. LIOTTA $\left({ }^{*}\right)$ and B. SILVESTRE-BRAC \\ Institut des Sciences Nucléaires, BP 257, 38044 Grenoble, France
}

(Reçu le 15 novembre 1977, accepté le 7 décembre 1977)

\begin{abstract}
Résumé. - Le traitement par la théorie du champ nucléaire du système à 3 particules est mené à tous les ordres de la théorie des perturbations sans toutefois inclure les excitations de trous. Il est montré que la théorie corrige proprement les violations du principe de Pauli ainsi que la redondance de la base.
\end{abstract}

Abstract. - The nuclear field treatment of the 3-particle system is carried out to all orders of perturbation theory but without including hole-excitations. It is shown that the theory properly corrects both the Pauli principle violations and the overcompleteness of the basis.

One of the main features of the nuclear field theory (NFT) is that it treats simultaneously the singleparticle and the pairing and particle-hole phonon degrees of freedom as elementary modes of excitation. The resulting violation of the Pauli principle as well as the non-orthogonality of the elementary modes is corrected within the formalism, at least when applied to exactly soluble models where the field diagrams can be summed up to all orders [1,2]. However, so far, it has not been possible to carry out such a summation in the general case due to the increasing complexity of the diagrammatic expansion with the order of perturbation in $1 / \Omega(\Omega=j+1 / 2)$.

In the present letter we consider a 3-particle system outside a closed shell core interacting through a two-body force. To analyze this problem in the framework of the NFT we will only consider pairing and single-particle degrees of freedom while the original fermion hamiltonian will be replaced by the nuclear field hamiltonian [3].

$$
\begin{aligned}
& \mathscr{H}_{\mathrm{f}}=\mathscr{H}_{\text {s.p. }}+\mathscr{H}_{\text {t.b. }}+\mathscr{H}_{\mathrm{b}}+\mathscr{H}_{\text {p.v. }} \\
& \mathcal{H}_{\text {s.p. }}=\sum_{j m} \varepsilon_{j} c_{j m}^{+} c_{j m} \\
& \mathscr{H}_{\mathrm{t.b.}}=\sum_{\lambda \mu} \sum_{\mathrm{p} \leqslant q} \sum_{r \leqslant s}\langle p q ; \lambda|V| r s ; \lambda\rangle \times \\
& \times P^{+}(p q ; \lambda \mu) P(r s ; \lambda \mu) \\
& \mathscr{H}_{\mathrm{b}}=\sum_{n \lambda \mu} \omega_{n}(\lambda) \Gamma_{n}^{+}(\lambda \mu) \Gamma_{n}(\lambda \mu) \\
& \mathscr{H}_{\mathrm{p} . v \mathrm{v}}=\sum_{n \lambda \mu} \sum_{r \leqslant s}\left\{\Lambda_{n}^{*}(r s ; \lambda) \Gamma_{n}^{+}(\lambda \mu) P(r s ; \lambda \mu)+\text { c.c. }\right\}
\end{aligned}
$$

where $\Gamma_{n}^{+}(\lambda \mu)$ is the pairing phonon creation operator, $\omega_{n}(\lambda)$ is the corresponding phonon energy, $\Lambda$ is the particle-vibration coupling constant given by

$$
\begin{aligned}
\Lambda_{n}(p q ; \lambda)=\sum_{r \leqslant s}\langle p q ; & \lambda|V| r s ; \lambda\rangle \times \\
& \times\left\langle n \lambda \mu\left|P^{+}(r s ; \lambda \mu)\right| 0\right\rangle^{*}
\end{aligned}
$$

and

$$
P^{+}(r s ; \lambda \mu)=\left(c_{r}^{+} c_{s}^{+}\right)_{\lambda \mu} / N(r, s)
$$

where

$$
N(r, s)=\left(1+\delta_{r, s}\right)^{1 / 2}
$$

We do not consider hole-excitations and therefore only TDA vertices are included, as shown in figure 1. The value of the phonon vertex $A$ is

$\left\langle 0\left|\left(c_{1}^{+} c_{2}^{+}\right)_{\lambda \mu}^{+} \mathcal{H}_{\text {p.v. }} \Gamma_{n}^{+}(\lambda \mu)\right| 0\right\rangle=N(1,2) \Lambda_{n}(12 ; \lambda)$

while the four-point vertex B is given by

$$
\begin{aligned}
& \left\langle 0\left|\left(c_{3}^{+} c_{4}^{+}\right)_{\lambda \mu}^{+} \mathcal{H}_{\mathrm{t} . \mathrm{b} .}\left(c_{1}^{+} c_{2}^{+}\right)_{\lambda \mu}\right| 0\right\rangle= \\
& \quad=N(1,2) N(3,4)\langle 34 ; \lambda|V| 12 ; \lambda\rangle .
\end{aligned}
$$

(*) Present address : Forskinginstitutet for Atomfysik - S 10405 Stockholm. 
The pairing field is determined, according to rule IV of the NFT [1], by the equation

$$
\begin{gathered}
\left(\omega_{n}(\lambda)-\left(\varepsilon_{1}+\varepsilon_{2}\right)\right)\langle n \lambda \mu| \\
=P_{r}^{+}(12 ; \lambda \mu)|0\rangle= \\
\langle 12 ; \lambda|V| r s ; \lambda\rangle \\
x\left\langle n \lambda \mu\left|P^{+}(r s ; \lambda \mu)\right| 0\right\rangle .
\end{gathered}
$$

Each state of the basic set $\left\{\alpha_{j} ; I\right\} \equiv\left\{n \lambda, i j_{i} ; I\right\}$ - where $\left\{n \lambda, i j_{i} ; I\right\}$ identifies the $n^{\text {th }}$ phonon of multipolarity $\lambda$ coupled to the $i^{\text {th }}$ particle of angular momentum $j_{i}$ to give the total angular momentum $I$ of the 3-particle system - contains only one pairing phonon and one single-particle state (rule I). The Brillouin-Wigner diagrammatic expansion of the

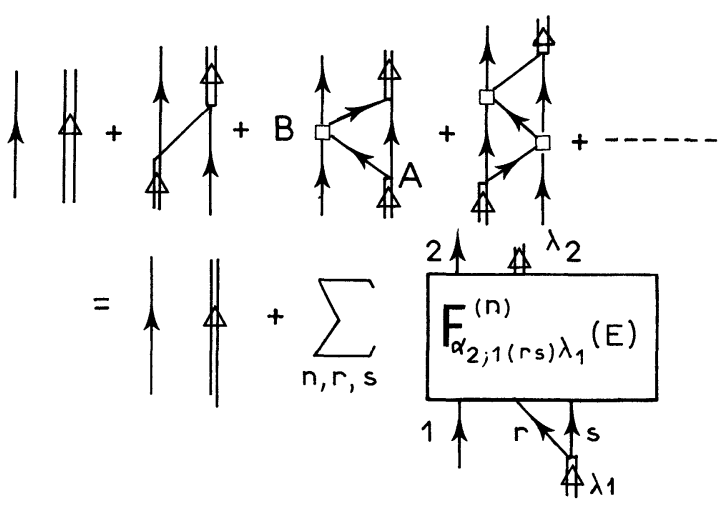

FIG. 1. - Nuclear field theory expansion of the effective interaction matrix elements $W_{\alpha_{2} \alpha_{1}}(E)$

effective interaction matrix element [4] $W_{\alpha_{2} \alpha_{1}}(E)$ shown in figure 1 (rule II) includes neither bubbles (rule III) nor any intermediate state which is also a basic state.

The zeroth order contribution to the matrix element $W$ is

$$
W_{\alpha_{2} \alpha_{1}}^{(0)}=\left(\varepsilon_{1}+\omega_{n_{1}}\left(\lambda_{1}\right)\right) \delta_{\alpha_{1}, \alpha_{2}} .
$$

To sum the whole diagrammatic series of figure 1 we notice that the contribution of a diagram of higher order $n$ can be written as

$$
W_{\alpha_{2} \alpha_{1}}^{(n)}(E)=\sum_{r s} F_{\alpha_{2} ; 1(r s) \lambda_{1}}^{(n)}(E) N(r, s) \Lambda_{n_{1}}\left(r s ; \lambda_{1}\right)
$$

where, in first order, the matrix element $F$ is given by

$$
\begin{aligned}
F_{\alpha_{2} ; 1(r s) \lambda_{1}}^{(1)}(E) & =N(1, r) \Lambda_{n_{2}}^{*}\left(r_{1} ; \lambda_{2}\right) \hat{\lambda}_{1} \hat{\lambda}_{2} \times \\
\times & \times\left\{\begin{array}{lll}
j_{s} & j_{r} & \lambda_{1} \\
j_{1} & I & \lambda_{2}
\end{array}\right\} \frac{1}{E-\left(\varepsilon_{1}+\varepsilon_{r}+\varepsilon_{s}\right)} \delta_{s, 2} .
\end{aligned}
$$

The total matrix element

$$
F_{\alpha_{2} ; 1(r s) \lambda_{1}}(E)=\sum_{n=1} F_{\alpha_{2} ; 1(r s) \lambda_{1}}^{(n)}(E)
$$

actually represents the propagator of the three particles which are originally in the intermediate state

$$
\left|1,(r s) \lambda_{1} ; I\right\rangle \text {. }
$$

Assuming that the propagator $F$ has been evaluated to order $n$, then one can evaluate the following order, $n+1$, making use of the diagram of figure 2 to get

$$
F_{\alpha 2 ; q(r s) \lambda}^{(n+1)}(E)=\sum_{r^{\prime} s^{\prime} \lambda^{\prime}} K_{q(r s) \lambda ; s\left(r^{\prime} s^{\prime}\right) \lambda^{\prime}}(E) F_{\alpha_{2} ; s\left(r^{\prime} s^{\prime}\right) \lambda^{\prime}}^{(n+)^{\prime}}(E)
$$

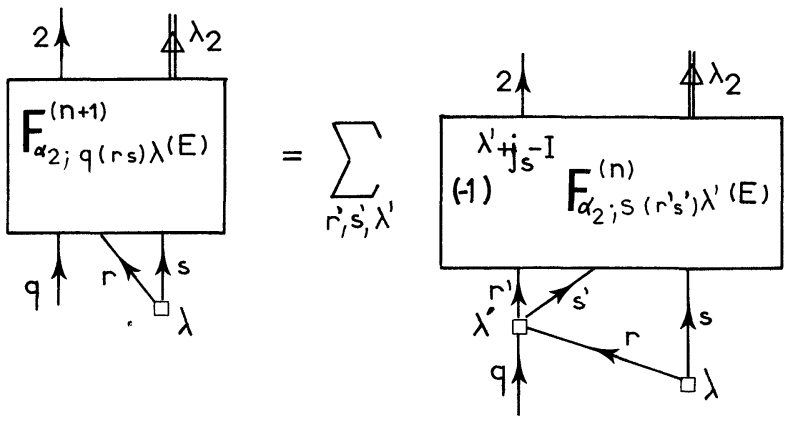

FIG. 2. - Graphical representation of the propagator $F$.

where

$$
\begin{gathered}
K_{q(r s) ; ; s\left(r^{\prime} s^{\prime}\right) \lambda}(E)=\hat{\lambda} \hat{\lambda}^{\prime}\left\{\begin{array}{lll}
j_{q} & j_{r} & \lambda^{\prime} \\
j_{s} & I & \lambda
\end{array}\right\} \frac{1}{E-\left(\varepsilon_{q}+\varepsilon_{r}+\varepsilon_{s}\right)} \\
x\left\langle r^{\prime} s^{\prime} ; \lambda^{\prime}|V| r q ; \lambda^{\prime}\right\rangle N\left(r^{\prime}, s^{\prime}\right) N(r, q) . \quad(8 b)
\end{gathered}
$$

Therefore the total propagator $F$ is given by

$$
\begin{aligned}
F_{\alpha_{2} ; q(r) \lambda}(E)= & F_{\alpha_{2} ; q(r s) \lambda}^{(1)}(E)+ \\
& +\sum_{r^{\prime} s^{\prime} \lambda^{\prime}} K_{q(r s) \lambda ; s\left(r^{\prime} s^{\prime}\right) \lambda^{\prime}}(E) F_{\alpha_{2} ; s\left(r^{\prime} s^{\prime}\right) \lambda^{\prime}}(E)
\end{aligned}
$$

which is an inhomogeneous set of energy dependent linear equations in the set of intermediate states

$$
\{|q,(r s) \lambda ; I\rangle\}
$$

for the propagator $F$. For each value of the final basic state $\alpha_{2}$ and as function of the energy $E$ one can solve eq. (9) to get the total TDA interaction matrix element as

$$
W_{\alpha_{2} \alpha_{1}}(E)=W_{\alpha_{2} \alpha_{1}}^{(0)}+\sum_{r s} N(r, s) \Lambda_{n_{1}}\left(r s ; \lambda_{1}\right) F_{\alpha_{2} ; 1(r s) \lambda_{1}}(E) .
$$
tion

The diagonalization of $W$ provides the wave func-

$$
|\alpha, I\rangle=\sum_{j} \xi_{\alpha_{j}}\left|\alpha_{j} ; I\right\rangle
$$

with the normalization condition [2]

$$
\sum_{i, j}\left\{\delta_{i j}-\frac{\partial}{\partial E} W_{\alpha_{i} \alpha_{j}}(E)\right\} \xi_{\alpha_{i}}^{*} \xi_{\alpha_{j}}=1 .
$$

The matrix elements of a given transition operator can be evaluated in the same way. For example, the evaluation of the one particle transfer matrix element $t\left(\beta_{j}, k, \alpha_{\mathrm{A}}\right)=\left\langle\beta_{j} ; I_{\mathrm{B}}\left|C_{k}^{+}\right| \alpha_{\mathrm{A}} ; I_{\mathrm{A}}\right\rangle$ between the state $\left|\alpha_{\mathrm{A}} ; I_{\mathrm{A}}\right\rangle$ of the target nucleus $\mathrm{A}$ and the 
basic states $\left\{\beta_{j}, I_{\mathrm{B}}\right\}$ that describe the physical state $\left|\beta_{\mathrm{B}}, I_{\mathrm{B}}\right\rangle$ of the residual nucleus $\mathrm{B}$ according to eq. (11), can be performed introducing the propagator $T$ of figure 3. Proceeding as for the propagator $F$
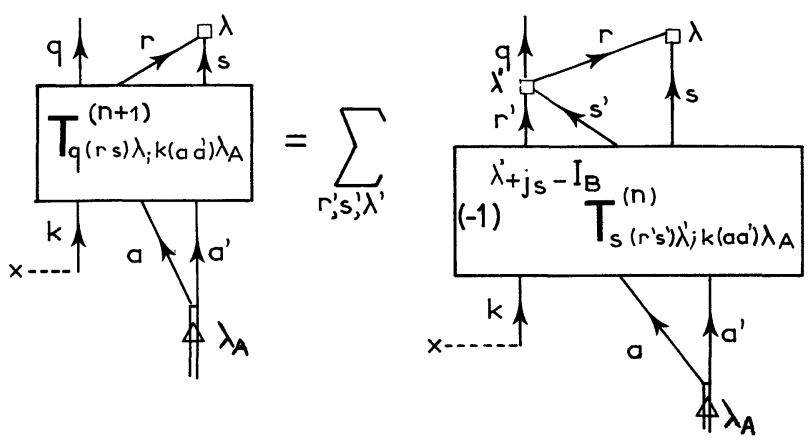

FIG. 3. - Graphical representation of the propagator $T$.

one obtains (notice that the energy $E_{\mathrm{B}}$ is now already known through the diagonalization of $W$ )

$$
\begin{aligned}
T_{q(r s) \lambda ; k\left(a a^{\prime}\right) \lambda_{\mathbf{A}}} & =T_{q(r s) \lambda ; k\left(a a^{\prime}\right) \lambda_{\mathbf{A}}}^{(1)}+\sum_{r^{\prime} \lambda^{\prime}} \hat{\lambda} \hat{\lambda}^{\prime}\left\{\begin{array}{ccc}
j_{q} & j_{r} & \lambda^{\prime} \\
j_{s} & I & \lambda
\end{array}\right\} \times \\
& \times\left\langle r q ; \lambda^{\prime}|V| r^{\prime} s^{\prime} ; \lambda^{\prime}\right\rangle N(r, q) N\left(r^{\prime}, s^{\prime}\right) \times \\
& \times \frac{1}{E_{\mathbf{B}}-\left(\varepsilon_{q}+\varepsilon_{r}+\varepsilon_{s}\right)} T_{s\left(r^{\prime} s^{\prime}\right) \lambda^{\prime} ; k\left(a a^{\prime}\right) \lambda_{\mathbf{A}}}
\end{aligned}
$$

which is also a set of inhomogeneous linear equations that provides - for each value of the intermediate state $\left|k\left(a a^{\prime}\right) \lambda_{\mathrm{A}} ; I\right\rangle$ - the propagator $T$. After solving the relatively simple energy independent eq. (13) the total TDA matrix element $t$ can be obtained as

$$
\begin{array}{r}
t\left(\beta_{j}, k, \alpha_{\mathrm{A}}\right)=\delta_{\left(n_{\mathrm{A}} \lambda_{\mathbf{A}}\right),\left(n_{\mathbf{B}} \lambda_{\mathbf{B}}\right)} \delta_{k, b}+\sum_{r s} N(r, s) \Lambda_{n_{\mathbf{B}}}^{*}\left(r s ; \lambda_{\mathrm{B}}\right) \times \\
\times \sum_{a a^{\prime}} T_{b(r s) \lambda_{\mathbf{B}} ; k\left(a a^{\prime}\right) \lambda_{\mathbf{A}}} N\left(a, a^{\prime}\right) \Lambda_{n_{\mathbf{A}}}\left(a a^{\prime}, \lambda_{\mathrm{A}}\right) \times \\
\times \frac{1}{E_{\mathrm{A}}-\left(\varepsilon_{a}+\varepsilon_{a^{\prime}}\right)}
\end{array}
$$

and the one-body transfer transition amplitude to the state $\left|\beta_{\mathbf{B}}, I_{\mathbf{B}}\right\rangle$ is given by

$$
S_{k}(\mathrm{~A} \rightarrow \mathrm{B})=\sum_{j} \xi_{\beta_{j}} t\left(\beta_{j}, k, \alpha_{\mathrm{A}}\right)
$$

As an illustration, we will consider here a single particle level model of spin $j$ and energy $\varepsilon$.

The total spin $I$ to be analyzed is such that only two values of $\lambda$ are allowed. In this simple case eq. (9), which is a $2 \times 2$ set of linear equations for each of the two basic states, can easily be analytically solved. The phonon energies are given by $\omega_{i}=2 \varepsilon+\left\langle j j ; \lambda_{i}|V| j j ; \lambda_{i}\right\rangle=2 \varepsilon+V_{i}$ and the coupling constants by $\Lambda_{n}\left(j j ; \lambda_{i}\right)=V_{i}$. After some algebra the matrix elements $W$ are found to have the values

$$
W_{i j}(E)=\left(\varepsilon+\omega_{i}\right) \delta_{i, j}+M_{i j}(E) / D(E)
$$

where, with

$$
\begin{aligned}
& \eta=(E-3 \varepsilon)^{-1} \text { and } C_{i j}=\left\{\begin{array}{lll}
j & j & \lambda_{i} \\
j & I & \lambda_{j}
\end{array}\right\}, \\
& M_{i j}(E)=2 V_{i} V_{j} \hat{\lambda}_{i} \hat{\lambda}_{j} C_{i j} \eta-\delta_{i, j} V_{i} Q \eta^{2}
\end{aligned}
$$

and

$$
\begin{aligned}
D(E) & =1-2\left(\hat{\lambda}_{1}^{2} V_{1} C_{11}+\hat{\lambda}_{2}^{2} V_{2} C_{22}\right) \eta+Q \eta^{2} \\
Q & =4 V_{1} V_{2} \hat{\lambda}_{1}^{2} \hat{\lambda}_{2}^{2}\left(C_{11} C_{22}-C_{12}^{2}\right) .
\end{aligned}
$$

The diagonalization of $W$ provides the equation

$$
A \eta^{2}-B \eta+1=0
$$

where

$A=V_{1} V_{2} \times$

$\times\left\{4 \hat{\lambda}_{1}^{2} \hat{\lambda}_{2}^{2}\left(C_{11} C_{22}-C_{12}^{2}\right)+2 \hat{\lambda}_{1}^{2} C_{11}+2 \hat{\lambda}_{2}^{2} C_{22}+1\right\}$

$B=V_{1}\left(2 \hat{\lambda}_{1}^{2} C_{11}+1\right)+V_{2}\left(2 \hat{\lambda}_{2}^{2} C_{22}+1\right)$.

The two values of $E$ given by eq. (19) may not always be physical. To analyze the situation in which both Pauli violating and overcomplete basic states are involved we first consider the case $I=3 j-4$ $\left(\lambda_{1}=2 j-1, \lambda_{2}=2 j-3\right)$ where no physical solution is allowed by the Pauli principle as can easily be checked by counting the allowed number of states in the m-scheme. Utilizing the Racah formula [5] one finds that in this case

$C_{11}=-1 /(8 j-2), \quad C_{12}=0, \quad C_{22}=-1 /(8 j-10)$

which implies $A=B=0$ and there is no solution to eq. (19).

Another interesting case is $I=3 j-3$ (also, here, $\lambda_{1}=2 j-1, \lambda_{2}=2 j-3$ ) where the only solution allowed by the Pauli principle has the value (cf. eqs. (26.45) and (26.11) of ref. [6])

$E=3 \varepsilon+3\left\{(j-1) V_{2}+(3 j-2) V_{1}\right\} /(4 j-3)$.

In this case one finds

$$
\begin{aligned}
& C_{11}=(5 j-3) /(2(4 j-1)(4 j-3)) \\
& C_{12}=-3 \times \\
& \times\{(3 j-2)(j-1) /(4 j-5)(4 j-1)\}^{1 / 2} /(2(4 j-3)), \\
& C_{22}=-j /(2(4 j-3)(4 j-5)) .
\end{aligned}
$$

which yields $A=0$ and the only solution given by (19) coincides with the exact result. Nevertheless, this only physical state is described by the NFT in terms of a two-dimensional overcomplete basis. Finally, it is worthwhile to point out that the NFT gives the correct answers in these cases through the non-trivial and general $6-j$ relations (21) and (22). In the above model, it is shown that the NFT, provided that it is carried out to all orders, gives exactly the results of the shell-model. We have some reasons to feel that this is a general feature of the theory also valid for more sophisticated cases. 


\section{References}

[1] BES, D. R. et al. Phys. Lett. 52B (1974) 253.

[2] Bes, D. R. et al. Nucl. Phys. A 260 (1976) 1.

For a general review of the NFT as well as references see Broglia, R. A., Bortignon, P. F., Bes, D. R. and Liotta, R. J., Phys. Rep. 30C (1977) 305.
[3] Bes, D. R. et al. Nucl. Phys. A 260 (1976) 77.

[4] Dussel, G. G. and Liotta, R. J., Phys. Lett. 37B (1971) 477.

[5] RaCaH, G., Phys. Rev. 62 (1942) 438.

[6] De Shalit, A. and TAlmi, I., Nuclear Shell Theory (Academic Press, New York and London), 1963. 